Article

\title{
Deformed Exponentials and Applications to Finance
}

\section{Barbara Trivellato}

Dipartimento di Scienze Matematiche "G.L. Lagrange", Politecnico di Torino, Corso Duca degli Abruzzi 24, 10129 Torino, Italy; E-Mail:barbara.trivellato@ polito.it; Tel.: +39-0110907550; Fax: +39-0110907599

Received: 29 July 2013; in revised form: 22 August 2013 / Accepted: 26 August 2013 /

Published: 2 September 2013

\begin{abstract}
We illustrate some financial applications of the Tsallis and Kaniadakis deformed exponential. The minimization of the corresponding deformed divergence is discussed as a criterion to select a pricing measure in the valuation problems of incomplete markets. Moreover, heavy-tailed models for price processes are proposed, which generalized the well-known Black and Scholes model.
\end{abstract}

Keywords: deformed logarithm; deformed exponential; generalized entropy; martingale measure; generalized Fokker-Plank equation; non-Gaussian option pricing

\section{Introduction}

In this paper, we illustrate some financial applications of the Tsallis [1] and Kaniadakis [2] one-parameter deformation of the ordinary logarithmic and exponential function. We introduce these deformed functions as examples of the more general definition of the $\varphi$-deformed exponential given by Naudts [3] (Chapter 10). Until now, the $\varphi$-exponential has mainly been used in statistical mechanics to generalize the standard Boltzmann-Gibbs mechanics. In particular, the Tsallis and Kaniadakis exponentials have proven to be suitable to explain a very large class of experimentally observed phenomena, which are described by distribution functions that exhibit power law tails, in low and high energy physics, but also in natural, social, financial and economic sciences; see e.g., [4-29].

It is well known in mathematical finance that any contingent claim can be replicated in a complete market, i.e., there exists a self-financing strategy and an initial capital (the replication price), so that the corresponding value of the portfolio at maturity equals, almost surely, the claim. The replication price, also called the arbitrage-free price, is the mean value of the discounted claim under the unique martingale (or risk-neutral) measure. In more realistic incomplete markets, however, a claim is not, 
in general, perfectly replicable. There is more than one martingale measure, equivalent to the basic probability measure, $\mathbb{P}$, which leads to different arbitrage-free contingent claim prices. Therefore, it is necessary to choose a criterion to select one specific equivalent martingale measure. Several criteria have been proposed in the literature to select a possibly $\mathbb{P}$-equivalent pricing martingale measure. Let us recall, among others, the minimal variance martingale measure (see e.g., [30]), the minimal martingale measure (see e.g., [31]) and the minimal entropy martingale measure (see, e.g., [32] and, for the problem of model calibration, [33]).

In mathematical finance, the Kaniadakis exponential was first introduced in [34] to define a new family of martingale measures, which are closely related to both the standard entropy martingale measure (see, e.g., [32]) and the well-known p-martingale measures (see, e.g., [35]), which are connected with the Tsallis exponential.

In this work, the $\varphi$-logarithm is used to introduce the notion of $\varphi$-divergence between two probability measures, which extends the standard Kullback-Leibler divergence. The minimization of this deformed divergence is then proposed as a general criterion to select a pricing measure in the valuation problems of incomplete markets. We address this issue specifically for the Tsallis and Kaniadakis logarithms.

There is a lot of empirical evidence in financial option pricing that suggests that models for equity (or indices) in which the underlying assets exhibit heavier tails than those of a log-normal distribution can provide a better fit to the real data of financial markets. This is the reason why many authors have tried to develop new models that go beyond the standard log-normal assumptions of the well-known Black and Scholes [36] model: local volatility models (see, e.g., [37,38]), stochastic volatility models (see, e.g., [39,40]) and jump-diffusion models (see, e.g., [41]). An interesting new approach was introduced by Borland [16,17], who described market dynamics through anomalously diffusing systems evolving in time according to a nonlinear Fokker-Plank equation. More precisely, she replaced the Wiener process in the Black and Scholes model by a process having a distribution, $f(t, x)$, which satisfies the nonlinear FP equation, $\frac{\partial}{\partial t} f(t, x)=\frac{1}{2} \frac{\partial^{2}}{\partial x^{2}} f^{1+\alpha}(t, x)$, with $-2 / 3<\alpha<0$. The solution of this equation is a Tsallis distribution with a nonlinear variance, $\sigma^{2}(t) \sim t^{1 /(2+\alpha)}$, and with a power law tail, $f(t, x) \sim x^{2 /(2+\alpha)}$; see, e.g., [42] and, in the general context of anomalously diffusing systems, [43]. In this paper, we illustrate how this approach can be generalized to include time-dependent distributions of the Tsallis and Kaniadakis type with a general time-dependent variance.

The paper is organized as follows. In Section 2, we give a general definition of the $\varphi$-deformed exponential and logarithm, and as examples, we introduce the Kaniadakis and Tsallis deformed exponentials and logarithms. The $\varphi$-deformed logarithm is used in Section 3 to generalize the standard notion of relative entropy or Kullback-Leibler divergence. We investigate the relationships between this relative entropy and the deformed Tsallis and Kaniadakis relative entropies, and we illustrate some applications of these entropies in finance. Particular attention is paid to the Kaniadakis entropy, which is relatively new in this field. In Section 4, we define the T-Gaussian and the K-Gaussian processes, whose heavy-tailed distributions solve some nonlinear time-dependent Fokker-Plank equations. These processes can be viewed as a generalization of the Wiener process, since their law is an extension of the Gaussian law based on the Kaniadakis and Tsallis exponentials, and we discuss some of their possible uses in the modeling of financial markets. 


\section{Deformed Exponentials}

The following definition of the $\varphi$-deformed exponential can be found in Naudts [3] (Chapter 10). He first defined the $\varphi$-deformed logarithm as:

$$
\ln _{\varphi}(x)=\int_{1}^{x} \frac{d t}{\varphi(t)}, \quad x>0
$$

where, denoting by $\mathbb{R}_{>}$the set of strictly positive real numbers, $\varphi: \mathbb{R}_{>} \rightarrow \mathbb{R}_{>}$is a strictly positive, nondecreasing and continuous function. As a consequence, the $\varphi$-logarithm:

$$
\ln _{\varphi}: \mathbb{R}_{>} \rightarrow\left(-\int_{0}^{1} \frac{d t}{\varphi(t)}, \int_{1}^{+\infty} \frac{d t}{\varphi(t)}\right)=(-m,+M)
$$

is a strictly increasing and concave function, satisfying $\ln _{\varphi}(1)=0$ and $(d / d x) \ln _{\varphi}(x)=1 / \varphi(x)$. In particular, $\ln _{\varphi}(x)$ is negative on $(0,1)$ and positive on $(1,+\infty)$. The natural logarithm is obtained with $\varphi(t)=t$.

The $\varphi$-logarithm is self-dual, namely $\ln _{\varphi}(x)=-\ln _{\varphi}(1 / x)$ holds true if and only if:

$$
\varphi(t)=t^{2} \varphi(1 / t), \quad \forall t>0
$$

With the change of variables, $t=1 / v$, in Equation (1), we in fact obtain:

$$
\int_{1}^{x} \frac{d t}{\varphi(t)}=-\int_{1}^{1 / x} \frac{d v}{v^{2} \varphi(1 / v)}
$$

If we let:

$$
\varphi(t)=t e^{-\theta(\ln t)}
$$

the condition in Equation (3) is satisfied, if and only if the real function, $\theta$, is even:

$$
\theta(y)=\theta(-y), \quad \forall y \in \mathbb{R}
$$

The transformation in Equation (5) suggests to us to give the following definition of the $\theta$-logarithm, equivalent to the definition of $\ln _{\varphi}$ :

$$
\ln _{\theta}(x)=\int_{0}^{\ln x} e^{\theta(y)} d y, \quad x>0
$$

where $\theta: \mathbb{R} \rightarrow \mathbb{R}$ is a continuous function, such that:

$$
y_{1} \leq y_{2} \Rightarrow \theta\left(y_{2}\right)-\theta\left(y_{1}\right) \leq y_{2}-y_{1}
$$

Let us observe that the assumption in Equation (8) on $\theta(y)$ is the counterpart of the assumption $\varphi(t)$ nondecreasing in the definition of $\ln _{\varphi}(x)$ and ensures that the $\theta$-deformed logarithm is concave. If $\theta(y)$ is differentiable, Equation (8) is equivalent to $(d / d y) \theta(y) \leq 1$. The $\theta$-deformed logarithm is self-dual, if and only if $\theta(y)$ is an even continuous function satisfying Equation (8). The ordinary logarithm is obtained with $\theta(y)=0$.

The $\varphi$-exponential is defined as the inverse function of $\ln _{\varphi}$ : 


$$
\exp _{\varphi}=\ln _{\varphi}^{-1}:(-m,+M) \rightarrow \mathbb{R}_{>}
$$

It is a positive, increasing and convex function, satisfying $\exp _{\varphi}(0)=1$ and $(d / d x) \exp _{\varphi}(x)=\varphi\left(\exp _{\varphi}(x)\right)$.

The $\varphi$-exponential is self-dual, namely $\exp _{\varphi}(x)=\left(\exp _{\varphi}(-x)\right)^{-1}$ holds true, if and only if the rate function:

$$
R(x)=\frac{\varphi\left(\exp _{\varphi}(x)\right)}{\exp _{\varphi}(x)}
$$

is even. We, in fact, get that:

$$
\frac{d}{d x}\left(\exp _{\varphi}(x) \exp _{\varphi}(-x)\right)=(R(x)-R(-x)) \exp _{\varphi}(x) \exp _{\varphi}(-x)
$$

Equivalently, $\exp _{\theta}(x)=\ln _{\theta}^{-1}(x)$ results in being the solution of the Cauchy problem:

$$
\left\{\begin{array}{l}
u^{\prime}(x)=u(x) e^{-\theta(\ln u(x))} \\
u(0)=1
\end{array}\right.
$$

and is self dual, if and only if $\theta(\ln u(x))=\theta(\ln u(-x))$.

We now give some examples of deformed logarithms and exponentials.

\subsection{Kaniadakis Logarithm and Exponential}

The Kaniadakis logarithm with parameter $\alpha \in[-1,1]$ is a deformed logarithm with $\varphi(t)=2 /\left(t^{\alpha-1}+t^{-\alpha-1}\right)$ or, equivalently, $\theta(y)=\ln (\cosh (\alpha y))$ by Equation (5), and $m=M=+\infty$ :

$$
\ln _{\alpha}^{\mathrm{K}}(x)=\left\{\begin{array}{lll}
\frac{x^{\alpha}-x^{-\alpha}}{2 \alpha}, & \text { if } & \alpha \neq 0 \\
\ln x, & \text { if } & \alpha=0
\end{array}\right.
$$

Since $\ln _{\alpha}^{\mathrm{K}}(x)=\ln _{-\alpha}^{\mathrm{K}}(x)$, it is possible to only consider $0 \leq \alpha \leq 1$. The inverse of the Kaniadakis logarithm is defined by:

$$
\begin{aligned}
\exp _{\alpha}^{\mathrm{K}}(x) & =\exp \left(\int_{0}^{x} \frac{d z}{\sqrt{1+\alpha^{2} z^{2}}}\right) \\
& =\left\{\begin{array}{lll}
\left(\alpha x+\sqrt{1+\alpha^{2} x^{2}}\right)^{\frac{1}{\alpha}}, & \text { if } & \alpha \neq 0 \\
\exp x, & \text { if } & \alpha=0
\end{array}\right.
\end{aligned}
$$

The Kaniadakis logarithm and exponential retain many of the features of the ordinary logarithm and exponential, respectively; in particular, they are self-dual. 


\subsection{Tsallis Logarithm and Exponential}

The Tsallis logarithm with parameter, $\alpha \leq 1$ is a deformed logarithm with $\varphi(t)=1 / t^{\alpha-1}$ or, equivalently, $\theta(y)=\alpha y$, and:

$$
m=\left\{\begin{array}{ll}
\frac{1}{\alpha}, & \text { if } \quad 0<\alpha \leq 1 \\
+\infty, & \text { if } \quad \alpha \leq 0
\end{array} \quad M=\left\{\begin{array}{lll}
+\infty, & \text { if } & 0 \leq \alpha \leq 1 \\
-\frac{1}{\alpha}, & \text { if } & \alpha<0
\end{array}\right.\right.
$$

and is defined by:

$$
\ln _{\alpha}^{\mathrm{T}}(x)=\left\{\begin{array}{lll}
\frac{x^{\alpha}-1}{\alpha}, & \text { if } & \alpha \leq 1, \quad \alpha \neq 0 \\
\ln x, & \text { if } \quad \alpha=0
\end{array}\right.
$$

Its inverse is defined by:

$$
\exp _{\alpha}^{\mathrm{T}}(x)= \begin{cases}(1+\alpha x)^{\frac{1}{\alpha}}, & \text { if } \quad(0<\alpha \leq 1, x>-1 / \alpha) \quad \text { or } \quad(\alpha<0, x<-1 / \alpha) \\ \exp x, & \text { if } \quad \alpha=0, \quad(x \in \mathbb{R})\end{cases}
$$

The Tsallis exponential can be extended to all of the real line by letting:

$$
\exp _{\alpha}^{\mathrm{T}}(x)= \begin{cases}0, & \text { if } \quad 0<\alpha \leq 1, x \leq-1 / \alpha \\ +\infty, & \text { if } \quad \alpha<0, x \geq-1 / \alpha\end{cases}
$$

Let us notice that the Tsallis logarithm and exponential are not self-dual.

Remark 1. The original definition of the Tsallis logarithm uses a different parametrization of $\alpha$ and, also, includes the case $\alpha>1$. Here, we have chosen the same parametrization for both the Tsallis and the Kaniadakis logarithms to make a comparison between them easier. The condition, $\alpha \leq 1$, is needed to make $\varphi(t)$ nondecreasing or, equivalently, $\theta(y)$, satisfying Equation (8), and, thus, $\ln _{\varphi}(x)$ is concave. Let us observe that the additional condition, $\alpha \geq 0$, ensures that $\ln _{\alpha}^{\mathrm{T}}(+\infty)=+\infty$.

\subsection{Other Examples}

The Nigel J. Newton logarithm [44,45] is a deformed logarithm with $\varphi(t)=\frac{t}{1+t}$ or, equivalently, $\theta(y)=\ln \left(1+e^{y}\right)$, and $m=M=+\infty$ :

$$
\ln ^{\mathrm{N}}(x)=x-1+\ln x
$$

The following deformed logarithm is obtained by taking $\varphi(t)=\frac{4}{t\left(t^{\alpha}-t^{-\alpha}\right)^{2}}, 0<\alpha \leq 1$; see [13,46]:

$$
\ln _{\varphi}(x)=\frac{1}{\alpha} \frac{x^{\alpha}-x^{-\alpha}}{x^{\alpha}+x^{-\alpha}}
$$

Its inverse is:

$$
\exp _{\varphi}(x)=\left(\frac{1+\alpha x}{1-\alpha x}\right)^{\frac{1}{2 \alpha}}, \quad-\frac{1}{\alpha}<x<\frac{1}{\alpha}
$$

and is connected to $\exp _{\alpha}^{\mathrm{K}}(x)$ through $\exp _{\varphi}(x)=\exp _{\alpha}^{\mathrm{K}}\left(\frac{x}{\sqrt{1-\alpha^{2} x^{2}}}\right)$. 


\section{Deformed Entropies}

Let $(\Omega, \mathcal{F}, \mathbb{P})$ be a probability space. Given another probability measure, $\mathbb{Q}$, on $(\Omega, \mathcal{F})$, the symbol, $\mathbb{Q} \ll \mathbb{P}$, stands for $\mathbb{Q}$ absolutely continuous with respect to (w.r.t.) $\mathbb{P}$, and $\frac{d \mathbb{Q}}{d \mathbb{P}}$ denotes the Radon-Nikodym derivative of $\mathbb{Q}$ w.r.t. $\mathbb{P}$.

Definition 1. Let $\mathbb{Q} \ll \mathbb{P}$ and $\Phi:(0,+\infty) \rightarrow \mathbb{P}$ be a convex function, $\Phi(0)=\lim _{x \rightarrow 0} \Phi(x)$. The $\Phi$-relative entropy of $\mathbb{Q}$ w.r.t. $\mathbb{P}$, or $\Phi$-divergence between $\mathbb{Q}$ and $\mathbb{P}$, is defined as:

$$
H_{\Phi}(\mathbb{Q} \mid \mathbb{P})=\mathbb{E}_{\mathbb{P}}\left[\Phi\left(\frac{d \mathbb{Q}}{d \mathbb{P}}\right)\right]
$$

provided that the integral in Equation (22) exists; otherwise, we let $H_{\Phi}(\mathbb{Q} \mid \mathbb{P})=+\infty$.

In the following, we assume that $\Phi$ is a continuous, strictly convex and differentiable function of the form, $\Phi(x)=x \ln _{\varphi}(x)$. Let us observe that the strict convexity of $\Phi(x)$ implies that $H_{\Phi}(\mathbb{Q} \mid \mathbb{P}) \geq \Phi(1)=0$, with equality, if and only if $\mathbb{P}=\mathbb{Q}$. Furthermore, the functional, $\mathbb{Q} \rightarrow H_{\Phi}(\mathbb{Q} \mid \mathbb{P})$, is strictly convex.

The standard relative entropy, $H_{0}(\mathbb{Q} \mid \mathbb{P})$, or Kullback-Leibler divergence, is defined by letting $\ln _{\varphi}(x)=\ln (x)$, while the Kaniadakis relative entropy, $H_{\alpha}^{\mathrm{K}}(\mathbb{Q} \mid \mathbb{P})$, is defined by letting $\ln _{\varphi}(x)=\ln _{\alpha}^{\mathrm{K}}(x)$. Applying the definition of $\ln _{\alpha}^{\mathrm{K}}$, we, in fact, obtain:

$$
H_{\alpha}^{\mathrm{K}}(\mathbb{Q} \mid \mathbb{P})=\frac{\mathbb{E}_{\mathbb{P}}\left[\left(\frac{d \mathbb{Q}}{d \mathbb{P}}\right)^{1+\alpha}-\left(\frac{d \mathbb{Q}}{d \mathbb{P}}\right)^{1-\alpha}\right]}{2 \alpha}
$$

From the definition of $\ln _{\alpha}^{\mathrm{K}}(x)$, for $\alpha=0, H_{\alpha}^{\mathrm{K}}(\mathbb{Q} \mid \mathbb{P})$ is the standard relative entropy, while for $\alpha=1$, it reduces to half of the variance of the Radon-Nikodym derivative:

$$
H_{1}^{\mathrm{K}}(\mathbb{Q} \mid \mathbb{P})=\frac{1}{2} \operatorname{Var}\left(\frac{d \mathbb{Q}}{d \mathbb{P}}\right)=\frac{1}{2} \mathbb{E}_{\mathbb{P}}\left[\left(\frac{d \mathbb{Q}}{d \mathbb{P}}\right)^{2}-1\right], \quad \text { if } \mathbb{Q} \ll \mathbb{P}
$$

Lemma 1. Let $\mathbb{Q} \ll \mathbb{P}$ and $\alpha \in(0,1]$. Then, $H_{\alpha}^{\mathrm{K}}(\mathbb{Q} \mid \mathbb{P})<+\infty$, if and only if $(d \mathbb{Q} / d \mathbb{P}) \in L^{1+\alpha}(\mathbb{P})$.

Proof. Since, for $0<\alpha<1,(d \mathbb{Q} / d \mathbb{P})^{1-\alpha}$ is concave, by Jensen inequality, we can deduce that $\mathbb{E}_{\mathbb{P}}\left[(d \mathbb{Q} / d \mathbb{P})^{1-\alpha}\right]<+\infty$. The conclusion follows from Equation (23).

The Tsallis relative entropy, $H_{\alpha}^{\mathrm{T}}(\mathbb{Q} \mid \mathbb{P})$, with $0 \leq \alpha \leq 1$, is defined by letting $\ln _{\varphi}(x)=\ln _{\alpha}^{\mathrm{T}}(x)$. By definition, for $\alpha=0$, it is the standard relative entropy, while for $0<\alpha \leq 1$, it is given by:

$$
H_{\alpha}^{\mathrm{T}}(\mathbb{Q} \mid \mathbb{P})= \begin{cases}\mathbb{E}_{\mathbb{P}}\left[\frac{\left(\frac{d \mathbb{Q}}{d \mathbb{P}}\right)^{1+\alpha}-1}{\alpha}\right], & \text { if } \mathbb{Q} \ll \mathbb{P} \\ +\infty, & \text { otherwise }\end{cases}
$$

In particular, for $\alpha=1$, it reduces to the variance of the Radon-Nikodym derivative:

$$
H_{1}^{\mathrm{T}}(\mathbb{Q} \mid \mathbb{P})=\operatorname{Var}\left(\frac{d \mathbb{Q}}{d \mathbb{P}}\right)=\mathbb{E}_{\mathbb{P}}\left[\left(\frac{d \mathbb{Q}}{d \mathbb{P}}\right)^{2}-1\right], \quad \text { if } \mathbb{Q} \ll \mathbb{P}
$$

Let us observe that $H_{0}^{\mathrm{T}}=H_{0}^{\mathrm{K}}=H_{0}$ and $H_{1}^{\mathrm{T}}=2 H_{1}^{\mathrm{K}}$. 
Remark 2. Since, for $\alpha>0,(d \mathbb{Q} / d \mathbb{P}) \in L^{1+\alpha}(\mathbb{P})$ is a sufficient condition for the standard relative entropy to be finite, from the previous lemma, we deduce that $H_{\alpha}^{\mathrm{K}}(\mathbb{Q} \mid \mathbb{P})<+\infty$ or, equivalently, $H_{\alpha}^{\mathrm{T}}(\mathbb{Q} \mid \mathbb{P})<+\infty$ implies that $H_{0}(\mathbb{Q} \mid \mathbb{P})<+\infty$.

The following proposition establishes the relationships between the standard, the Kaniadakis and the Tsallis relative entropies.

Proposition 1. Let $\alpha \in(0,1]$. Then:

$$
H_{0}(\mathbb{Q} \mid \mathbb{P})-c_{\alpha} \leq H_{\alpha}^{\mathrm{K}}(\mathbb{Q} \mid \mathbb{P}) \leq H_{\alpha}^{\mathrm{T}}(\mathbb{Q} \mid \mathbb{P})
$$

where the positive constant, $c_{\alpha}$, goes to zero as $\alpha \rightarrow 0$.

Proof. The first inequality follows from the fact that $\ln (x) \leq \ln _{\alpha}(x)$ for $x \geq 1$, and the converse inequality holds true if $x \leq 1$. In particular:

$$
\begin{aligned}
& H_{\alpha}^{\mathrm{K}}(\mathbb{Q} \mid \mathbb{P})=\mathbb{E}_{\mathbb{P}}\left[\frac{d \mathbb{Q}}{d \mathbb{P}} \ln _{\alpha}\left(\frac{d \mathbb{Q}}{d \mathbb{P}}\right)\right] \geq \int_{\frac{d \mathbb{Q}}{d \mathbb{P}}>1}\left[\frac{d \mathbb{Q}}{d \mathbb{P}} \ln \left(\frac{d \mathbb{Q}}{d \mathbb{P}}\right)\right] d \mathbb{P}+\int_{\frac{d \mathbb{Q}}{d \mathbb{P}} \leq 1}\left[\frac{d \mathbb{Q}}{d \mathbb{P}} \ln _{\alpha}\left(\frac{d \mathbb{Q}}{d \mathbb{P}}\right)\right] d \mathbb{P} \\
& =H_{0}(\mathbb{Q} \mid \mathbb{P})+\int_{\frac{d \mathbb{Q}}{d \mathbb{P}} \leq 1}\left[\frac{d \mathbb{Q}}{d \mathbb{P}}\left(\ln _{\alpha}\left(\frac{d \mathbb{Q}}{d \mathbb{P}}\right)-\ln \left(\frac{d \mathbb{Q}}{d \mathbb{P}}\right)\right)\right] d \mathbb{P} \geq H_{0}(\mathbb{Q} \mid \mathbb{P})-c_{\alpha}
\end{aligned}
$$

where:

$$
c_{\alpha}=\max _{x \in[0,1]}\left(x \ln (x)-x \ln _{\alpha}(x)\right)
$$

The second inequality follows from:

$$
H_{\alpha}^{\mathrm{K}}(\mathbb{Q} \mid \mathbb{P})=\frac{1}{2} H_{\alpha}^{\mathrm{T}}(\mathbb{Q} \mid \mathbb{P})+\frac{1}{2} H_{-\alpha}^{\mathrm{T}}(\mathbb{Q} \mid \mathbb{P})
$$

and:

$$
H_{-\alpha}^{\mathrm{T}}(\mathbb{Q} \mid \mathbb{P}) \leq H_{0}(\mathbb{Q} \mid \mathbb{P}) \leq H_{\alpha}^{\mathrm{T}}(\mathbb{Q} \mid \mathbb{P})
$$

where we defined:

$$
H_{-\alpha}^{\mathrm{T}}(\mathbb{Q} \mid \mathbb{P})=\frac{\mathbb{E}_{\mathbb{P}}\left[\frac{d \mathbb{Q}}{d \mathbb{P}}\right]^{1-\alpha}-1}{-\alpha}
$$

In fact, the Kaniadakis logarithmic can be expressed in terms of the Tsallis logarithmic as follows:

$$
\ln _{\alpha}^{\mathrm{K}}(x)=\frac{1}{2} \ln _{\alpha}^{\mathrm{T}}(x)+\frac{1}{2} \ln _{-\alpha}^{\mathrm{T}}(x)
$$

which implies Equation (30).

Using $\ln (x) \leq x-1$ and Jensen inequality, we obtain:

$$
\begin{gathered}
H_{-\alpha}^{\mathrm{T}}(\mathbb{Q} \mid \mathbb{P})=\frac{\mathbb{E}_{\mathbb{P}}\left[\frac{d \mathbb{Q}}{d \mathbb{P}}\right]^{1-\alpha}-1}{-\alpha} \leq \frac{1}{-\alpha} \ln \left(\mathbb{E}_{\mathbb{P}}\left[\frac{d \mathbb{Q}}{d \mathbb{P}}\right]^{1-\alpha}\right) \\
\leq \mathbb{E}_{\mathbb{P}}\left[\frac{d \mathbb{Q}}{d \mathbb{P}} \ln \frac{d \mathbb{Q}}{d \mathbb{P}}\right]=H_{0}(\mathbb{Q} \mid \mathbb{P})
\end{gathered}
$$


which is the first inequality of Equation (31). On the other hand, using similar arguments we get:

$$
\begin{gathered}
H_{\alpha}^{\mathrm{T}}(\mathbb{Q} \mid \mathbb{P})=\frac{\mathbb{E}_{\mathbb{P}}\left[\frac{d \mathbb{Q}}{d \mathbb{P}}\right]^{1+\alpha}-1}{\alpha} \geq \frac{1}{\alpha} \ln \left(\mathbb{E}_{\mathbb{P}}\left[\frac{d \mathbb{Q}}{d \mathbb{P}}\right]^{1+\alpha}\right) \\
\geq \mathbb{E}_{\mathbb{P}}\left[\frac{d \mathbb{Q}}{d \mathbb{P}} \ln \frac{d \mathbb{Q}}{d \mathbb{P}}\right]=H_{0}(\mathbb{Q} \mid \mathbb{P})
\end{gathered}
$$

which is the second inequality of Equation (31).

\section{1. $\Phi-P r o j e c t i o n s$}

Let us denote by $\mathcal{K}$ a convex set of probability measures on $(\Omega, \mathcal{F})$, which are absolutely continuous w.r.t. $\mathbb{P}$, and define $\mathcal{K}_{\Phi}=\left\{\mathbb{Q} \in \mathcal{K}: H_{\Phi}(\mathbb{Q} \mid \mathbb{P})<+\infty\right\}$.

Definition 2. A measure, $\mathbb{Q}^{*} \in \mathcal{K}$, is called a $\Phi$-projection of $\mathbb{P}$ on $\mathcal{K}$ if:

$$
H_{\Phi}\left(\mathbb{Q}^{*} \mid \mathbb{P}\right)=\inf _{\mathbb{Q} \in \mathcal{K}} H_{\Phi}(\mathbb{Q} \mid \mathbb{P})=: H_{\Phi}(\mathcal{K} \mid \mathbb{P})
$$

We recall some relevant results about $\Phi$-projections:

1. If $\Phi$ is strictly convex and $H_{\Phi}(\mathcal{K} \mid \mathbb{P})<+\infty$, then there exists at most one $\Phi$-projection of $\mathbb{P}$ on $\mathcal{K}$ (see, e.g., Proposition 8.2 in [47]).

2. If $\mathcal{K}$ is closed in the variational distance topology and $\lim _{x \rightarrow+\infty} \frac{\Phi(x)}{x}=+\infty$, then there exists a $\Phi$-projection of $\mathbb{P}$ on $\mathcal{K}$ (see, e.g., Proposition 8.5 in [47]).

Let us observe that, if $\ln _{\varphi}(+\infty)=+\infty$, as for the standard, the Tsallis $(0 \leq \alpha \leq 1)$ and the Kaniadakis logarithms, then $\Phi(x)=x \ln _{\varphi}(x)$ satisfies the equality, $\lim _{x \rightarrow+\infty} \frac{\Phi(x)}{x}=+\infty$.

3. Let $\mathbb{Q}^{*} \in \mathcal{K}_{\Phi}$. Then, $\mathbb{Q}^{*}$ is the $\Phi$-projection of $\mathbb{P}$ on $\mathcal{K}$, if and only if the directional derivative of $H_{\Phi}(\cdot \mid \mathbb{P})$ in $\mathbb{Q}^{*}$ is non-negative along any direction from $\mathbb{Q}^{*}$ to any $\mathbb{Q} \in \mathcal{K}_{\Phi}$, (see [48]):

$$
\int \Phi^{\prime}\left(\frac{d \mathbb{Q}^{*}}{d \mathbb{P}}\right)\left(d \mathbb{Q}^{*}-d \mathbb{Q}\right) \leq 0, \quad \forall \mathbb{Q} \in \mathcal{K}_{\Phi}
$$

4. Let $\mathbb{Q}^{*} \in \mathcal{K}_{\Phi}$. Under some additional growth conditions on $\Phi(x)$ —see [47]—it holds that $\mathbb{Q}^{*}$ is the $\Phi$-projection of $\mathbb{P}$ on $\mathcal{K}$, if and only if $\Phi^{\prime}\left(\frac{d \mathbb{Q}^{*}}{d \mathbb{P}}\right) \in L^{1}(\mathbb{Q})$ and $\mathbb{E}_{\mathbb{Q}^{*}}\left[\Phi^{\prime}\left(\frac{d \mathbb{Q}^{*}}{d \mathbb{P}}\right)\right] \leq$ $\mathbb{E}_{\mathbb{Q}}\left[\Phi^{\prime}\left(\frac{d \mathbb{Q}^{*}}{d \mathbb{P}}\right)\right], \forall \mathbb{Q} \in \mathcal{K}_{\Phi}$

A crucial issue, for example, in mathematical finance, is to know whether the $\Phi$-projection, $\mathbb{Q}^{*}$, of $\mathbb{P}$ on $\mathcal{K}$ is equivalent to $\mathbb{P}$ (in symbols, $\mathbb{Q}^{*} \sim \mathbb{P}$ ). Frittelli [32] studied the minimal entropy martingale measure, which corresponds to the $\Phi$-projection when $\Phi(x)=x \ln (x)$ and $\mathcal{K}$ is a suitable class of martingale probability measures on which the $\Phi$-projection exists. In this case, he showed that the minimal entropy martingale measure is automatically equivalent to $\mathbb{P}$ if a measure, $\mathbb{Q} \in \mathcal{K}_{\Phi}$, equivalent to $\mathbb{P}$ exists (see, also, the analogue result by Csizar [49]). On the base of the point 3 above, this fact can be extended to general $\Phi$-projections. 
Corollary 1. Let $\Phi^{\prime}(0)=-\infty$. Assume a measure, $\mathbb{Q} \in \mathcal{K}_{\Phi}$, with $\mathbb{Q} \sim \mathbb{P}$, exists. If $\mathbb{Q}^{*}$ is the $\Phi$ projection of $\mathbb{P}$ on $\mathcal{K}$, then $\mathbb{Q}^{*} \sim \mathbb{P}$.

Proof. Suppose that $\mathbb{Q}^{*}$ is not equivalent to $\mathbb{P}$, that is, $\mathbb{P}\left(\frac{d \mathbb{Q}^{*}}{d \mathbb{P}}=0\right)>0$. Since $\mathbb{Q} \sim \mathbb{P}$, we deduce that $\mathbb{Q}\left(\frac{d \mathbb{Q}^{*}}{d \mathbb{P}}=0\right)>0$; but, under the hypothesis, $\Phi^{\prime}(0)=-\infty$, this leads to a contradiction to the necessary condition on a $\Phi$-projection of the point 3 above.

Let us observe that, to prove the corollary above, we have employed $\Phi^{\prime}(0)=-\infty$. This condition is fulfilled by $\Phi(x)=x \ln _{\alpha}^{\mathrm{K}}(x)$ if $\alpha$ is strictly less than one, but not by $\Phi(x)=x \ln _{\alpha}^{\mathrm{T}}(x)$, for all $0<\alpha \leq 1$, where it holds, $\Phi^{\prime}(0)=-1 / \alpha$.

The Kaniadakis projection has been investigated in [34] in the same financial framework as [32]. The existence of the Kaniadakis projection has been derived from the points 1 and 2 above, and its equivalence to $\mathbb{P}$ from Corollary 1. Furthermore, using the point 4 above, some characterizations of its density w.r.t. $\mathbb{P}$ have been provided. These results, which hold for $0 \leq \alpha<1$, extend those given in [32] for $\alpha=0$.

More precisely, let $X=\left(X_{t}\right)_{0 \leq t \leq T}$ be a locally bounded $\mathbb{R}^{d}$-valued semimartingale defined on a filtered probability space, $\left(\Omega, \mathcal{F}, \mathbb{F}=\left(\mathcal{F}_{t}\right)_{0 \leq t \leq T}, \mathbb{P}\right)$, where the filtration, $\mathbb{F}$, satisfies the usual conditions, $\mathcal{F}_{0}$ is trivial, $\mathcal{F}=\mathcal{F}_{T}$ and $T \in(0,+\infty)$ is a fixed time horizon. $X$ represents the discounted asset prices in an incomplete financial market. Let $\mathcal{M}$ be the set of local martingale measures, that is, the set of all $\mathbb{P}$-absolutely continuous probability measures that turn $X$ into a local martingale, and let $\mathcal{M}^{e}$ be the subset of $\mathcal{M}$ consisting of $\mathbb{P}$-equivalent martingale measures. Moreover, define:

$$
\mathcal{M}_{\alpha}=\left\{\mathbb{Q} \in \mathcal{M}: H_{\alpha}^{\mathrm{K}}(\mathbb{Q} \mid \mathbb{P})<+\infty\right\}, \quad \mathcal{M}_{\alpha}^{e}=\mathcal{M}_{\alpha} \cap \mathcal{M}^{e}
$$

In the following, we let $\Phi_{\alpha}(x)=x \ln _{\alpha}^{\mathrm{K}}(x)$, and we denote by $\mathbb{Q}_{\alpha}^{\mathrm{K}}$ the Kaniadakis projection of $\mathbb{P}$ on $\mathcal{M}$, called the minimal K-entropy martingale measure. Moreover, we assume $0 \leq \alpha<1$.

\section{Theorem 1.}

1. If $\mathcal{M}_{\alpha} \neq \emptyset$, then $\mathbb{Q}_{\alpha}^{\mathrm{K}}$ exists.

2. If $\mathcal{M}_{\alpha}^{e} \neq \emptyset$, then $\mathbb{Q}_{\alpha}^{\mathrm{K}}$ exists and is equivalent to $\mathbb{P}$.

Theorem 2. Let $\overline{\mathbb{Q}} \in \mathcal{M}_{\alpha}^{e}$. Then, $\overline{\mathbb{Q}}=\mathbb{Q}_{\alpha}^{\mathrm{K}}$, if and only if:

1. $\frac{d \overline{\mathbb{Q}}}{d \mathbb{P}}=\left(\Phi_{\alpha}^{\prime}\right)^{-1}(\beta-g)$, where $\beta \in \mathbb{R}, g \in L^{1}(\overline{\mathbb{Q}}), \mathbb{E}_{\overline{\mathbb{Q}}}[g]=0$ and

$$
\left(\Phi_{\alpha}^{\prime}\right)^{-1}(x)=\exp _{\alpha}^{\mathrm{K}}\left(\frac{x-\sqrt{1-\alpha^{2}+\alpha^{2} x^{2}}}{1-\alpha^{2}}\right)
$$

2. $g \in \mathcal{C}_{\alpha}=\left\{f \in \bigcap_{\mathbb{Q} \in \mathcal{M}_{\alpha}} L^{1}(\mathbb{Q}): \mathbb{E}_{\mathbb{Q}}[f] \leq 0, \forall \mathbb{Q} \in \mathcal{M}_{\alpha}\right\}$.

Theorem 3. Let $\overline{\mathbb{Q}} \in \mathcal{M}_{\alpha}^{e}$. Then, $\overline{\mathbb{Q}}=\mathbb{Q}_{\alpha}^{\mathrm{K}}$, if and only if:

1. $\frac{d \overline{\mathbb{Q}}}{d \mathbb{P}}=\left(\Phi_{\alpha}^{\prime}\right)^{-1}\left(\beta-\left(\int \eta d X\right)_{T}\right)$, where $\beta \in \mathbb{R}, \eta$ is $X$-integrable;

2. $\mathbb{E}_{\mathbb{Q}}\left[\left(\int \eta d X\right)_{T}\right]=0$ for $\mathbb{Q}=\overline{\mathbb{Q}}, \mathbb{Q}_{\alpha}^{\mathrm{K}}$. 
The proof of the theorems above can be found in [34].

The Tsallis projection of $\mathbb{P}$ on $\mathcal{M}$, which we denote by $\mathbb{Q}_{\alpha}^{\mathrm{T}}$, has been studied in mathematical finance by several authors; see, e.g., [35]. In fact, it has been introduced as the optimal solution of the equivalent problem of minimizing the $L^{p}$-norm of $\frac{d \mathbb{Q}}{d \mathbb{P}}$, with $p>1$. For this reason, it is known as the $p$-optimal martingale measure. In particular, the point 2 of Theorem 1 has been proven in [50] when the price process, $X$, is continuous. Let us observe that, from Remark 2, we deduce that, for $0<\alpha \leq 1$ :

$$
\left\{\mathbb{Q} \in \mathcal{M}: H_{\alpha}^{\mathrm{T}}(\mathbb{Q} \mid \mathbb{P})<+\infty\right\}=\left\{\mathbb{Q} \in \mathcal{M}: H_{\alpha}^{\mathrm{K}}(\mathbb{Q} \mid \mathbb{P})<+\infty\right\}=\mathcal{M}_{\alpha}
$$

The following result follows from Proposition 1 and $\mathcal{M}_{0} \subseteq \mathcal{M}_{\alpha}$ (see Remark 2).

Proposition 2. Let:

$$
V_{0}=\inf _{\mathbb{Q} \in \mathcal{M}_{0}} H_{0}(\mathbb{Q} \mid \mathbb{P}), \quad V_{\alpha}^{\mathrm{K}}=\inf _{\mathbb{Q} \in \mathcal{M}_{\alpha}} H_{\alpha}^{\mathrm{K}}(\mathbb{Q} \mid \mathbb{P}), \quad V_{\alpha}^{\mathrm{T}}=\inf _{\mathbb{Q} \in \mathcal{M}_{\alpha}} H_{\alpha}^{\mathrm{T}}(\mathbb{Q} \mid \mathbb{P})
$$

If $V_{\alpha}^{\mathrm{T}} \rightarrow V_{0}$ as $\alpha \rightarrow 0$, then $V_{\alpha}^{\mathrm{K}} \rightarrow V_{0}$ as $\alpha \rightarrow 0$.

Remark 3. Conditions on the convergence of $V_{\alpha}^{\mathrm{T}}$ to $V_{0}$ as $\alpha \rightarrow 0$ can be found in, e.g., [51].

In the next section, we explicitly compute the Kaniadakis and Tsallis projections in a simple one-period finite state market model. We show that the Kaniadakis projection interpolates the minimal entropy martingale measure and the minimal variance martingale measure, which are two well known $\Phi$-projections in mathematical finance. Moreover, we show that there are situations in which the Tsallis projection is only $\mathbb{P}$-absolutely continuous, while the corresponding Kaniadakis projection is equivalent to $\mathbb{P}$.

\subsection{Example in a One-Period Finite State Market Model}

Let $n \geq 2$ be the cardinality of $\Omega, r$ be one plus the interest rate and $\mathcal{T}=\{0,1\}$ be the set of times. Denote the risky asset we consider by $S=\left(S_{0}, S_{1}\right)$ and assume that $S_{0}=1$ and that $S_{1}$ takes $n$ different positive values, $a(i), i=1, \ldots, n$, with probability, $\mathbb{P}\left(S_{1}=a(i)\right)=p(i)>0$. Consider the problem:

$$
\min _{q \in \mathcal{M}}\left(\sum_{i=1}^{n} q(i) \ln _{\alpha}^{\mathrm{K}}\left(\frac{q(i)}{p(i)}\right)\right)
$$

where $\mathcal{M}=\left\{q \in \mathbb{R}^{n}, q \geq 0, \sum_{i=1}^{n} q(i)=1, \sum_{i=1}^{n} q(i) a(i)=r\right\}$ is the convex set of risk neutral (or martingale) probability measures. Using the method of Lagrange multipliers, for $0<\alpha<1$, the solution of Equation (41) is given by:

$$
q_{\alpha}^{\mathrm{K}}(i)=p(i)\left(\Phi_{\alpha}^{\prime}\right)^{-1}(-\lambda-\gamma a(i))
$$

where $\Phi_{\alpha}(x)=x \ln _{\alpha}^{\mathrm{K}}(x)$ and $(\lambda, \gamma) \in \mathbb{R}^{2}$ is the unique solution of the following two equations:

$$
\left\{\begin{array}{l}
\sum_{i=1}^{n} p(i)\left(\Phi_{\alpha}^{\prime}\right)^{-1}(-\lambda-\gamma a(i))=1 \\
\sum_{i=1}^{n} p(i) a(i)\left(\Phi_{\alpha}^{\prime}\right)^{-1}(-\lambda-\gamma a(i))=r
\end{array}\right.
$$


For $\alpha=0$, the solution of Equation (41) is the minimal entropy martingale measure given by (see [32]):

$$
q^{e n t}(i)=q_{0}^{\mathrm{K}}(i)=\frac{p(i) e^{-\gamma a(i)}}{\sum_{i=1}^{n} p(i) e^{-\gamma a(i)}}
$$

where $\gamma \in \mathbb{R}$ is the unique solution of the following equation:

$$
\sum_{i=1}^{n} p(i)(a(i)-r) e^{-\gamma a(i)}=0
$$

For $\alpha=1$, the solution of Equation (41) is the minimal variance martingale measure and is given by:

$$
q^{v a r}(i)=q_{1}^{\mathrm{K}}(i)=p(i)\left(1+\frac{\mu-r}{\sigma^{2}}(\mu-a(i))\right)
$$

where $\mu=\sum_{i=1}^{n} p(i) a(i), \sigma^{2}=\sum_{i=1}^{n} p(i)(a(i))^{2}-\mu^{2}$.

In the following numerical example, we compare $q^{\text {ent }}, q^{v a r}$ and $q_{\alpha}^{\mathrm{K}}$, for different values of $\alpha$.

Let $n=3, r=1, a(1)=1+(x+2) / 4, a(2)=1+x / 4, a(3)=1+(x-2) / 4$ and $x \in \mathbb{R}$, and assume $p(1)=p(3)=1 / 4, p(2)=1 / 2$. It can be checked that a measure, $q \in \mathcal{M}$, with $q>0$ (and therefore, equivalent to $p$ ) exists, if and only if $|x|<2$. Under this assumption, we have already noticed in Corollary 1 of the previous section that $q^{e n t}$ and, for all $0<\alpha<1, q_{\alpha}^{\mathrm{K}}$ are always equivalent to $p$, while $q^{v a r}$ is equivalent to $p$, if and only if $|x|<1$. From Equations (44) and (45), the minimal entropy martingale measure is given by:

$$
q^{e n t}(1)=\frac{(2-x)^{2}}{16}, \quad q^{e n t}(2)=\frac{4-x^{2}}{8}, \quad q^{\text {ent }}(3)=\frac{(2+x)^{2}}{16}
$$

From Equation (46), the minimal variance martingale measure is given by:

$$
q^{v a r}(1)=\frac{1}{4}(1-x), \quad q^{v a r}(2)=1-q^{v a r}(1)-q^{v a r}(3), \quad q^{v a r}(3)=\frac{1}{4}(1+x)
$$

The solution $(\lambda, \gamma)$ of Equation (43), computed through a numerical procedure for $x=0, \pm 1 / 2, \pm 1$, $\pm 3 / 2$ and $\alpha=1 / 4,1 / 2,3 / 4$, is reported in Table 1 .

Table 1. Solution $(\lambda, \gamma)$ of Equation (43) computed for different choices of $x$ and $\alpha$.

\begin{tabular}{lccc}
\hline$(\lambda, \gamma)$ & $\alpha=1 / 4$ & $\alpha=1 / 2$ & $\alpha=3 / 4$ \\
\hline$x=-3 / 2$ & $(2.28049,-3.95826)$ & $(2.34499,-4.15981)$ & $(2.45742,-4.50550))$ \\
$x=-1$ & $(0.93836,-2.21744)$ & $(0.94162,-2.27150)$ & $(0.91767,-2.32605)$ \\
$x=-1 / 2$ & $(-0.04312,-1.02406)$ & $(-0.05019,-1.02886)$ & $(-0.07087,-1.02751)$ \\
$x=0$ & $(-1,0)$ & $(-1,0)$ & $(-1,0)$ \\
$x=+1 / 2$ & $(-2.09125,1.02406)$ & $(-2.10792,1.02886)$ & $(-2.12589,1.02751)$ \\
$x=+1$ & $(-3.49652,2.21744)$ & $(-3.60138,2.27150)$ & $(-3.73442,2.32604)$ \\
$x=+3 / 2$ & $(-5.63603,3.95826)$ & $(-5.97463,4.15981)$ & $(-6.55358,4.50549)$ \\
\hline
\end{tabular}

A comparison between $q^{\text {ent }}, q^{v a r}$ and, for $\alpha=1 / 4,1 / 2,3 / 4, q_{\alpha}^{\mathrm{K}}$, shows that:

$$
q^{v a r}(1)=q_{1}^{\mathrm{K}}(1) \leq q_{3 / 4}^{\mathrm{K}}(1) \leq q_{1 / 2}^{\mathrm{K}}(1) \leq q_{1 / 4}^{\mathrm{K}}(1) \leq q_{0}^{\mathrm{K}}(1)=q^{e n t}(1)
$$




$$
\begin{aligned}
& q^{e n t}(2)=q_{0}^{\mathrm{K}}(2) \leq q_{1 / 4}^{\mathrm{K}}(2) \leq q_{1 / 2}^{\mathrm{K}}(2) \leq q_{3 / 4}^{\mathrm{K}}(2) \leq q_{1}^{\mathrm{K}}(2)=q^{\text {var }}(2) \\
& q^{\text {var }}(3)=q_{1}^{\mathrm{K}}(3) \leq q_{3 / 4}^{\mathrm{K}}(3) \leq q_{1 / 2}^{\mathrm{K}}(3) \leq q_{1 / 4}^{\mathrm{K}}(3) \leq q_{0}^{\mathrm{K}}(3)=q^{\text {ent }}(3)
\end{aligned}
$$

It can be seen that $q^{\text {ent }}$ gives a higher probability to the extreme states than $q^{v a r}$, while the converse is true for the middle state. The Kaniadakis projection, $q_{\alpha}^{\mathrm{K}}$, results in being a modulation of $q^{\text {ent }}$ and $q^{v a r}$, and the value of $\alpha$ moves $q_{\alpha}^{\mathrm{K}}$ from one extreme measure to the other.

Now, let us consider the same entropy minimization problem as Equation (41), but with the Tsallis logarithm:

$$
\min _{q \in \mathcal{M}}\left(\sum_{i=1}^{n} q(i) \ln _{\alpha}^{\mathrm{T}}\left(\frac{q(i)}{p(i)}\right)\right)
$$

For $0<\alpha<1$, the solution of Equation (52) is given by:

$$
q_{\alpha}^{\mathrm{T}}(i)=p(i)\left(\Psi_{\alpha}^{\prime}\right)^{-1}(-\lambda-\gamma a(i))
$$

where $\Psi_{\alpha}(x)=x \ln _{\alpha}^{\mathrm{T}}(x),\left(\Psi_{\alpha}^{\prime}\right)^{-1}(y)=\exp _{\alpha}^{\mathrm{T}}(y) / \exp _{\alpha}^{\mathrm{T}}(1)$ and $(\lambda, \gamma) \in \mathbb{R}^{2}$ satisfies:

$$
\left\{\begin{array}{l}
\sum_{i=1}^{n} p(i)\left(\Psi_{\alpha}^{\prime}\right)^{-1}(-\lambda-\gamma a(i))=1 \\
\sum_{i=1}^{n} p(i) a(i)\left(\Psi_{\alpha}^{\prime}\right)^{-1}(-\lambda-\gamma a(i))=r
\end{array}\right.
$$

For $\alpha=0$ and $\alpha=1$, the solution of Equation (52) is $q^{\text {ent }}$ and $q^{v a r}$, respectively.

Let us assume again that $n=3, r=1, a(1)=1+(x+2) / 4, a(2)=1+x / 4, a(3)=1+(x-2) / 4$ and $x \in \mathbb{R}$. We know that, under the assumption, $|x|<2$, $q_{\alpha}^{\mathrm{K}}$ is equivalent to $p$, for all $0<\alpha<1$. Now, let us choose $x=-3+\sqrt{17} \in(1,2)$, so that $a(2) a(3)=1$, and for simplicity in computation, let $k=1 / 2$. Furthermore, let us assume $p(i)>0(i=1,2,3), \sum_{i=1}^{3} p(i)=1$ and $p(2)=4 p(3) a(3)$ (equivalently, $4 p(3)=p(2) a(2)$ ). Therefore, $p(2)=\frac{4}{4+a(2)}(1-p(1)), p(3)=\frac{a(2)}{4+a(2)}(1-p(1))$. It can be checked that $(\lambda, \gamma)=\left(3 \sqrt{\frac{4+a(2)}{(1+a(2))(1-p(1))}},-\lambda a(1)+\frac{1}{\kappa}\right)$ is the unique solution of Equation (54). This implies that the solution of Equation (52) is $q_{1 / 2}^{\mathrm{T}}(1)=0, q_{1 / 2}^{\mathrm{T}}(2)=\frac{1}{1+a(2)}$ and $q_{1 / 2}^{\mathrm{T}}(3)=\frac{a(2)}{1+a(2)}$, which is not equivalent to $p$.

\section{Deformed Gaussian Processes}

In this section, we define two stochastic processes, which can be viewed as a generalization of the Wiener process, since their law is an extension of the Gaussian law based on the Kaniadakis and Tsallis exponential. Thanks to the tail properties of these deformed exponentials, such processes can be used in finance to generalize the Black and Scholes option pricing model, providing a better fit to the real data of financial markets than that obtained by using processes with normal law.

The centered K-Gaussian probability density of parameters $\alpha \in(0,1]$ and $\sigma>0$ is defined as:

$$
f_{\alpha}^{\mathrm{K}}(x)=\left(Z_{\alpha}^{\mathrm{K}}\right)^{-1} \exp _{\alpha}^{\mathrm{K}}\left(-\frac{1}{2 \sigma^{2}} x^{2}\right), \quad x \in \mathbb{R}
$$

where $\left(Z_{\alpha}^{\mathrm{K}}\right)^{-1}$ is the normalization factor. Since: 


$$
\int_{0}^{+\infty} x^{r-1} \exp _{\alpha}^{\mathrm{K}}(-x) d x=\frac{1}{(2 \alpha)^{r}(1+r \alpha)} \frac{\Gamma\left(\frac{1}{2 \alpha}-\frac{r}{2}\right)}{\Gamma\left(\frac{1}{2 \alpha}+\frac{r}{2}\right)} \Gamma(r)
$$

where $r \alpha<1$ and $\Gamma(z)=\int_{0}^{\infty} e^{-t} t^{z-1} d t$ is the Gamma function (see formula (A20) in [13]), it results that:

$$
\left(Z_{\alpha}^{\mathrm{K}}\right)^{-1}=\frac{1}{\sqrt{\pi} \sigma} \sqrt{\alpha}\left(1+\frac{\alpha}{2}\right) \frac{\Gamma\left(\frac{1}{2 \alpha}+\frac{1}{4}\right)}{\Gamma\left(\frac{1}{2 \alpha}-\frac{1}{4}\right)}
$$

Unlike the standard case, from Equation (56), we can deduce that the K-Gaussian has finite moments only under some suitable choices of the parameter, $\alpha$; for example, the variance is finite if $\alpha<2 / 3$. Explicitly:

$$
\int_{\mathbb{R}} x^{2} f_{\alpha}^{\mathrm{K}}(t, x) d x=\Gamma_{\alpha} \sigma^{2}
$$

where:

$$
\Gamma_{\alpha}=\frac{\left(1+\frac{\alpha}{2}\right) \Gamma\left(\frac{1}{2 \alpha}+\frac{1}{4}\right) \Gamma\left(\frac{1}{2 \alpha}-\frac{3}{4}\right)}{2 \alpha\left(1+\frac{3}{2} \alpha\right) \Gamma\left(\frac{1}{2 \alpha}-\frac{1}{4}\right) \Gamma\left(\frac{1}{2 \alpha}+\frac{3}{4}\right)}
$$

is an increasing function of $\alpha(0<\alpha<2 / 3)$. When $\alpha$ goes to zero, the $\mathrm{K}$-Gaussian distribution approaches the usual centered Gaussian distribution with variance $\sigma^{2}$, while for larger $\alpha$, it shows fat tails, which vanish according to a power law.

Let $\sigma(t)$ be any differentiable function from $\mathbb{R}_{>}$to $\mathbb{R}_{>}$, possibly depending on $\alpha$. Define:

$$
f_{\alpha}^{\mathrm{K}}(t, x)=\left(Z_{\alpha}^{\mathrm{K}}(t)\right)^{-1} \exp _{\alpha}^{\mathrm{K}}\left(-\frac{1}{2 \sigma^{2}(t)} x^{2}\right)
$$

where $\left(Z_{\alpha}^{\mathrm{K}}(t)\right)^{-1}$ is given by Equation (57) after replacing $\sigma$ with $\sigma(t)$.

Proposition 3. $f_{\alpha}^{\mathrm{K}}(t, x)$ given by Equation (60) solves the nonlinear time-dependent Kolmogorov Forward, or Fokker-Plank, equation:

$$
\begin{aligned}
\frac{\partial f(t, x)}{\partial t} & =\frac{1}{2} \sigma(t) \dot{\sigma}(t) \frac{\partial}{\partial x}\left[\left(\left(Z_{\alpha}^{\mathrm{K}}(t) f(t, x)\right)^{\alpha}+\left(Z_{\alpha}^{\mathrm{K}}(t) f(t, x)\right)^{-\alpha}\right) \frac{\partial f(t, x)}{\partial x}\right] \\
& =\frac{1}{2} \sigma(t) \dot{\sigma}(t) \frac{\partial^{2}}{\partial x^{2}}\left[\frac{\left(Z_{\alpha}^{\mathrm{K}}(t)\right)^{\alpha}}{1+\alpha} f(t, x)^{1+\alpha}+\frac{\left(Z_{\alpha}^{\mathrm{K}}(t)\right)^{-\alpha}}{1-\alpha} f(t, x)^{1-\alpha}\right]
\end{aligned}
$$

Proof. It suffices to substitute the expression in Equation (60) of $f_{\alpha}^{\mathrm{K}}(t, x)$ into the Fokker-Plank equation. In fact, thanks to property $\exp _{\alpha}^{\mathrm{K}}(x) \exp _{\alpha}^{\mathrm{K}}(-x)=1$ and by the definition of $\exp _{\alpha}^{\mathrm{K}}$, we get:

$$
\begin{aligned}
& \left(Z_{\alpha}^{\mathrm{K}}(t) f_{\alpha}^{\mathrm{K}}(t, x)\right)^{\alpha}+\left(Z_{\alpha}^{\mathrm{K}}(t) f_{\alpha}^{\mathrm{K}}(t, x)\right)^{-\alpha}=\left(\exp _{\alpha}^{\mathrm{K}}\left(-\frac{1}{2 \sigma^{2}(t)} x^{2}\right)\right)^{\alpha}+\left(\exp _{\alpha}^{\mathrm{K}}\left(\frac{1}{2 \sigma^{2}(t)} x^{2}\right)\right)^{\alpha} \\
& \quad=2 \phi(t, x)
\end{aligned}
$$

where $\phi(t, x)=\sqrt{1+\alpha^{2} \frac{x^{4}}{4 \sigma^{4}(t)}}$. Since $\frac{\partial f_{\alpha}^{\mathrm{K}}(t, x)}{\partial x}=-\frac{1}{\sigma^{2}(t)} x \frac{f_{\alpha}^{\mathrm{K}}(t, x)}{\phi(t, x)}$, the right-hand side of the Fokker-Plank equation reduces to: 


$$
-\frac{\dot{\sigma}(t)}{\sigma(t)} \frac{\partial}{\partial x}\left[x f_{\alpha}^{\mathrm{K}}(t, x)\right]=-\frac{\dot{\sigma}(t)}{\sigma(t)}\left(1-\frac{x^{2}}{\sigma^{2}(t) \phi(t, x)}\right) f_{\alpha}^{\mathrm{K}}(t, x)
$$

We now check that Equation (63) equals the left-hand side of the Fokker-Plank equation. In fact:

$$
\frac{\partial f_{\alpha}^{\mathrm{K}}(t, x)}{\partial t}=-\left(\frac{\dot{Z}_{\alpha}^{\mathrm{K}}(t)}{Z_{\alpha}^{\mathrm{K}}(t)}-\frac{x^{2}}{\sigma^{2}(t) \phi(t, x)} \frac{\dot{\sigma}(t)}{\sigma(t)}\right) f_{\alpha}^{\mathrm{K}}(t, x)
$$

By the definition of $Z_{\alpha}^{\mathrm{K}}(t)$, we get:

$$
\frac{\dot{Z}_{\alpha}^{\mathrm{K}}(t)}{Z_{\alpha}^{\mathrm{K}}(t)}=\frac{\dot{\sigma}(t)}{\sigma(t)}
$$

Substituting these expressions in Equation (64), we can deduce the equality between Equations (64) and (63).

Remark 4. It has been proven in [52] that the K-Gaussian is not an exact solution of the purely $\mathrm{K}-$ diffusive equation:

$$
\frac{\partial f(t, x)}{\partial t}=\frac{D}{2} \frac{\partial^{2}}{\partial x^{2}}\left(f(t, x)^{1+\alpha}+f(t, x)^{1-\alpha}\right)
$$

where $D$ is a constant diffusion coefficient, but it requires an additional time-dependent term. This can be seen from the Fokker-Plank equation of Proposition 3, because of the presence of the two different powers, $\left(Z_{\alpha}^{\mathrm{K}}(t)\right)^{\alpha}$ and $\left(Z_{\alpha}^{\mathrm{K}}(t)\right)^{-\alpha}$, which, multiplied by $\sigma(t) \dot{\sigma}(t)$, cannot be constant simultaneously.

The centered T-Gaussian probability density of parameters $\alpha \in(-2,0)$ and $\sigma>0$ is defined as:

$$
f_{\alpha}^{\mathrm{T}}(x)=\left(Z_{\alpha}^{\mathrm{T}}\right)^{-1} \exp _{\alpha}^{\mathrm{T}}\left(-\frac{1}{2 \sigma^{2}} x^{2}\right), \quad x \in \mathbb{R}
$$

where:

$$
\left(Z_{\alpha}^{\mathrm{T}}\right)^{-1}=\frac{1}{\sqrt{2 \pi} \sigma} \sqrt{-\alpha} \frac{\Gamma\left(-\frac{1}{\alpha}\right)}{\Gamma\left(-\frac{1}{\alpha}-\frac{1}{2}\right)}
$$

Let us note that, for $\alpha \leq-2$, the distribution cannot be normalized, while for $\alpha>0$, it vanishes outside the interval, $\left(-\frac{\sqrt{2}}{\alpha} \sigma, \frac{\sqrt{2}}{\alpha} \sigma\right)$. Moreover, it can be proven that the variance of the T-Gaussian distribution is finite if $-2 / 3<\alpha<0$. As for the K-Gaussian distribution, when $\alpha$ goes to zero, the T-Gaussian distribution approaches the usual centered Gaussian distribution, with variance $\sigma^{2}$, while for larger $|\alpha|$, it shows fatter tails than the Gaussian distribution, since it decays with a power law in $|x|$ instead of exponentially.

Define:

$$
f_{\alpha}^{\mathrm{T}}(t, x)=\left(Z_{\alpha}^{\mathrm{T}}(t)\right)^{-1} \exp _{\alpha}^{\mathrm{T}}\left(-\frac{1}{2 \sigma^{2}(t)} x^{2}\right)
$$

where $\left(Z_{\alpha}^{\mathrm{T}}(t)\right)^{-1}$ is given by Equation (68) after replacing $\sigma$ with $\sigma(t)$. 
Proposition 4. $f_{\alpha}^{\mathrm{T}}(t, x)$ given by Equation (69) solves the nonlinear time-dependent Fokker-Plank equation:

$$
\begin{aligned}
\frac{\partial f(t, x)}{\partial t} & =\sigma(t) \dot{\sigma}(t) \frac{\partial}{\partial x}\left[\left(Z_{\alpha}^{\mathrm{T}}(t) f(t, x)\right)^{\alpha} \frac{\partial f(t, x)}{\partial x}\right] \\
& =\sigma(t) \dot{\sigma}(t) \frac{\partial^{2}}{\partial x^{2}}\left[\frac{\left(Z_{\alpha}^{\mathrm{T}}(t)\right)^{\alpha}}{1+\alpha} f(t, x)^{1+\alpha}\right]
\end{aligned}
$$

Proof. Similarly to the proof of Proposition 3, by substituting the expression in Equation (69) of $f_{\alpha}^{\mathrm{T}}(t, x)$ into the Fokker-Plank equation, we obtain that both the right-hand side and the left-hand side of the equation equals:

$$
-\frac{\dot{\sigma}(t)}{\sigma(t)}\left(1-\frac{x^{2}}{\sigma^{2}(t) \phi(t, x)}\right) f_{\alpha}^{\mathrm{T}}(t, x)
$$

where $\phi(t, x)=1-\frac{\alpha x^{2}}{2 \sigma^{2}(t)}$.

The purely T-diffusive Fokker-Plank equation:

$$
\frac{\partial f(t, x)}{\partial t}=\frac{D}{2} \frac{\partial^{2}}{\partial x^{2}} f(t, x)^{1+\alpha}
$$

has been widely studied in the literature; see, e.g., [42,43]. Solving the equation:

$$
\sigma(t) \dot{\sigma}(t) \frac{\left(Z_{\alpha}^{\mathrm{T}}(t)\right)^{\alpha}}{1+\alpha}=\frac{D}{2}
$$

leads to $\sigma(t) \sim t^{1 /(2+\alpha)}$. In finance, the Fokker-Plank Equation (72) has been used to model the market dynamics through anomalous diffusions; see, e.g., [16-19,22]. Indeed, it suggests to define the process:

$$
d \Omega_{t}=g\left(t, \Omega_{t}\right) d W_{t}
$$

where $W_{t}$ is the Wiener process and:

$$
g\left(t, \Omega_{t}\right)=\sqrt{D\left(f_{\alpha}^{\mathrm{T}}\left(t, \Omega_{t}\right)\right)^{\alpha}}
$$

Letting $\Omega_{0}=0$ and $\sigma^{2}(t) \equiv \sigma_{\alpha}^{2}(t) \rightarrow t$ as $\alpha$ goes to zero, Equation (74) defines a "generalized Wiener process" with a Tsallis distribution at each time, $t$, defined by Equation (69). The process, $\Omega_{t}$, has been then used to define the dynamics of the asset price process, $X$, as:

$$
d X_{t}=\mu X_{t} d t+\sigma X_{t} d \Omega_{t}
$$

where $\mu$ and $\sigma$ are constant parameters. The reason for the use of the process, $\Omega_{t}$, instead of the standard Wiener process, as in the Black and Scholes model, relies on the fact that models that exhibit heavier tails than those with log-normal distribution may provide a better fit for many equities (or indexes).

We are extending the model in Equation (76) in a working paper to consider the more general case, where $\Omega_{t}$ is described by the time-dependent nonlinear Fokker-Plank equation of Proposition 3 or 4 . In the first case: 


$$
g\left(t, \Omega_{t}\right)=\sqrt{\sigma(t) \dot{\sigma}(t)\left(\frac{\left(Z_{\alpha}^{\mathrm{K}}(t) f_{\alpha}^{\mathrm{K}}\left(t, \Omega_{t}\right)\right)^{\alpha}}{1+\alpha}+\frac{\left(Z_{\alpha}^{\mathrm{K}}(t) f_{\alpha}^{\mathrm{K}}\left(t, \Omega_{t}\right)\right)^{-\alpha}}{1-\alpha}\right)}
$$

while in the second:

$$
g\left(t, \Omega_{t}\right)=\sqrt{2 \sigma(t) \dot{\sigma}(t) \frac{\left(Z_{\alpha}^{\mathrm{T}}(t) f_{\alpha}^{\mathrm{T}}\left(t, \Omega_{t}\right)\right)^{\alpha}}{1+\alpha}}
$$

Let us observe that, in both cases, if $f_{\alpha}^{\mathrm{K}}\left(t, \Omega_{t}\right)$ or $f_{\alpha}^{\mathrm{T}}\left(t, \Omega_{t}\right)$ is small, then $g\left(t, \Omega_{t}\right)$ is large. Consequently, an unlikely step for $\Omega_{t}$ tends to be followed by a large jump. Since the analytical expression of $\sigma(t)$ is not a priori specified, as for the purely T-diffusive case, our pricing models are suitable to describe different types of (possibly, non-linear) variance changes with time. Obviously, the types of smile and skew patterns that can be generated within this framework determine how well these models fit real data. The downside of this approach is that it is no longer possible to have closed-form formulas for European option prices, like in the Black and Scholes model. However, we can still calculate option prices by using some numerical methods, for example, based on discretization of equations or Monte Carlo simulations; see, e.g., [53-55].

\section{Conflicts of Interest}

The author declares no conflict of interest.

\section{References}

1. Tsallis, C. Possible generalization of Boltzmann-Gibbs statistics. J. Stat. Phys. 1988, 52, 479-487.

2. Kaniadakis, G. Non-linear kinetics underlying generalized statistics. Phys. A: Stat. Mech. Appl. 2001, 296, 405-425.

3. Naudts, J. Generalised Thermostatistics; Springer: London, UK, 2011.

4. Curado, E.M.F; Tsallis, C. Generalized statistical mechanics connection with thermodynamics. J. Phys. A 1991, 24, L69-L72.

5. Tsallis, C. What are the numbers that experiments provide?. Quimica Nova 1994, 17, 468-471.

6. Tsallis, C.; Lévy, S.V.F.; Souza, A.M.C.; Maynard, R. Statistical-mechanical foundation of the ubiquity of Lévy distributions in nature. Phys. Rev. Lett. 1995, 75, 3589-3593.

7. Tsallis, C. Generalized entropy-based criterion for consistent testing. Phys. Rev. E 1998, 58, 1442-1445.

8. Tsallis, C.; Mendes, R.S.; Plastino, A.R. The role of constraints within generalized nonextensive statistics. Phys. A: Stat. Mech. Appl. 1998, 261, 534-554.

9. Tsallis, C. Introduction to Nonextensive Statistical Mechanics: Approaching a Complex World; Springer: Berlin, Germany, 2009.

10. Kaniadakis, G. H-theorem and generalized entropies within the framework of nonlinear kinetics. Phys. Lett. A 2001, 288, 283-291.

11. Kaniadakis, G. Statistical mechanics in the context of special relativity. Phys. Rev. E 2002, 66, 056125 . 
12. Kaniadakis, G.; Scarfone, A.M. Lesche stability of kappa-entropy. Phys. A: Stat. Mech. Appl. 2004, 340, 102-109.

13. Kaniadakis, G. Statistical mechanics in the context of special relativity II. Phys. Rev. E 2005, 72, 036108.

14. Kaniadakis, G. Maximum entropy principle and power-law tailed distributions. Eur. Phys. J. B 2009, 70, 3-13.

15. Pistone, G. א-exponential models from the geometrical viewpoint. Eur. Phys. J. B 2009, 70, 29-37.

16. Borland, L. A theory of non-Gaussian option pricing. Quant. Financ. 2002, 2, 415-431.

17. Borland, L. Option pricing formulas based on a non-Gaussian stock price model. Phys. Rev. Lett. 2002, $89,098701$.

18. Michael, F.; Johnson, M.D. Financial market dynamics. Phys. A: Stat. Mech. Appl. 2003, 320, 525-534.

19. Borland, L.; Bouchaud, J.P. A non-Gaussian option pricing model with skew. Quant. Financ. 2004, 4 , 499-514.

20. Rajaonarison, D.; Bolduc, D.; Jayet, H. The K-deformed multinomial logit model. Econ. Lett. 2005, 86, 13-20.

21. D. Rajaonarison. Deterministic heterogeneity in tastes and product differentiation in the K-logit model. Econ. Lett. 2008, 100, 396-398.

22. Vellekoop, M.; Nieuwenhuis, H. On option pricing models in the presence of heavy tails. Quant. Financ. 2007, 7, 563-573.

23. Clementi, F.; Gallegati, M; Kaniadakis G. kappa-Generalized statistics in personal income distribution. Eur. Phys. J. B 2007, 52 , 187-193.

24. Clementi, F; Di Matteo, T; Gallegati, M; Kaniadakis, G. The kappa-generalized distribution: A new descriptive model for the size distribution of incomes. Phys. A: Stat. Mech. Appl. 2008, 387, 3201-3208.

25. Clementi, F; Gallegati, M; Kaniadakis, G. A kappa-generalized statistical mechanics approach to income analysis. J. Stat. Mech. 2009, 02, P02037.

26. Clementi, F.; Gallegati, M.; Kaniadakis, G. A model of personal income distribution with application to Italian data. Empir. Econ. 2010, 39, 559-591.

27. Clementi, F.; Gallegati, M; Kaniadakis, G. A new model of income distribution: the k-generalized distribution. J. Econ. 2012, 105 , 63-91.

28. Bertotti, M.L.; Modanese, G. Exploiting the flexibility of a family of models for taxation and redistribution. Eur. Phys. J. B 2012, 85, 1-10.

29. Tapiero, O.J. A maximum (non-extensive) entropy approach to equity options bid-ask spread. Phys. A: Stat. Mech. Appl. 2013, 392, 3051-3060.

30. Föllmer, H.; Sondermann, D. Hedging of non-redundant contingent Claims. In Contributions to Mathematical Economics; Hildenbrand, W. and Mas-Colell, A. Eds.; North-Holland: Amsterdam, The Netherlands, 1986; Chapter 12.

31. Föllmer, H.; Schweizer, M. Hedging of contingent claims under incomplete information. In Applied Stochastic Analysis; Stochastics Monographs, vol. 5; Davis, M.H.A. and Elliot, R.J. Eds.; Gordon and Breach: London, UK, 1991; pp. 389-414. 
32. Frittelli, M. The minimal entropy martingale measure and the valuation problem in incomplete markets. Math. Financ. 2000, 10, 39-52.

33. Avellaneda, M. Minimum-relative-entropy calibration of asset-pricing models. Int. J. Theor. Appl. Finance 1998 , 01, 447-472.

34. Trivellato, B. The minimal $\kappa$-entropy martingale measure. Int. J. Theor. Appl. Finance 2012, 15, 1250038 .

35. Grandits, $\mathrm{P}$. The $p$-optimal martingale measure and its asymptotic relation with the minimal entropy martingale measure. Bernoulli 1999, 5, 225-247.

36. Black, F.; Scholes, M. The pricing of options and corporate liabilities. J. Polit. Econ. 1973, 81, 637-654.

37. Cox, J.C.; Ross, S.A. The valuation of options for alternative stochastic processes. J. Financ. Econ. 1976, $3,145-166$.

38. Dupire, B. Pricing with a smile. Risk Mag. 1994, 7, 19-20.

39. Hull, J; White, A. The pricing of options on assets with stochastic volatilities. J. Financ. 1987, 42, 281-300.

40. Heston, S.L. A closed-form solution for options with stochastic volatility with applications to bond and currency options. Rev. Financ. Stud. 1993, 6, 327-343.

41. Andersen, L.; Andreasen, J. Jump-diffusion processes: Volatility smile fitting and numerical methods for pricing. Rev. Deriv. Res. 2000, 4, 231-262.

42. Plastino, A.R.; Plastino, A. Non-extensive statistical mechanics and generalized Fokker-Plank equation. Phys. A: Stat. Mech. Appl. 1995, 222, 347-354.

43. Tsallis, C.; Bukman D.J. Anomalous diffusion in the presence of external forces: Exact timedependent solutions and their thermostatistical basis. Phys. Rev. 1996, 54, R2197.

44. Newton, N.J. An infinite-dimensional statistical manifold modelled on Hilbert space. J. Funct. Anal. 2012, 263, 1661-1681.

45. Pistone, G. Nonparametric information geometry. 2013, arXiv:1306.0480

46. Vigelis, R.F.; Cavalcante, C.C. On the $\varphi$-family of probability distributions. J. Theor. Probab. 2013, 26, 870-884.

47. Liese, F.; Vajda, I. Convex Statistical Distances; Teubner: Leipzig , Germany, 1987.

48. Rüschendorf, L. On the minimum discrimination information theorem. Statistics and Decisions 1984, $1,263-283$.

49. Csizár, I. I-divergence geometry of probability distributions and minimization problems. Ann. Probab. 1975, 3, 146-158.

50. Grandits, P.; Krawczyk, L. Closedness of some spaces of stochastic integrals. In Lecture Notes in Mathematics; Volume 1686; Springer: Berlin, Germany, 1998; pp. 73-85.

51. Santacroce, M. On the convergence of the $p$-optimal martingale measures to the minimal entropy martingale measure. Stoch. Anal. Appl. 2005, 23, 31-54.

52. Wada, T. and Scarfone, A.M. Asymptotic solutions of a nonlinear equation in the framework of $\kappa$-generalized statistical mechanics. Eur. Phys. J. B 2009, 70, 65-71.

53. D'Ippoliti, F; Moretto, E.; Pasquali, S.; Trivellato, B. Exact pricing with stochastic volatility and jumps. Int. J. Theor. Appl. Finance 2010, 13, 901-929. 
54. D’Ippoliti, F; Moretto, E.; Pasquali, S.; Trivellato, B. Exact and approximated option pricing in a stochastic volatility jump-diffusion model. In Mathematical and Statistical Methods for Actuarial Sciences and Finance ; Volume XII; Springer: Milan, Italy, 2010; pp. 103-112.

55. Moretto, E.; Pasquali, S.; Trivellato, B. Derivative evaluation using recombining trees under stochastic volatility. Adv. Appl. Stat. Sci. 2010, 1, 453-480.

(c) 2013 by the author; licensee MDPI, Basel, Switzerland. This article is an open access article distributed under the terms and conditions of the Creative Commons Attribution license (http://creativecommons.org/licenses/by/3.0/). 